\title{
Trends in suicidal behaviour and use of mental health services in Canadian military and civilian populations
}

\author{
Jitender Sareen MD, Tracie O. Afifi PhD, Tamara Taillieu MSc, Kristene Cheung MA, Sarah Turner BHSc, \\ Shay-Lee Bolton MSc, Julie Erickson MA, Murray B. Stein MD MPH, Deniz Fikretoglu PhD, \\ Mark A. Zamorski MD MHSA
}

\begin{abstract}
- Abstract
Background: In the context of the Canadian mission in Afghanistan, substantial media attention has been placed on mental health and lack of access to treatment among Canadian Forces personnel. We compared trends in the prevalence of suicidal behaviour and the use of mental health services between Canadian military personnel and the general population from 2002 to 2012/13.

Methods: We obtained data for respondents aged 18-60 years who participated in 4 nationally representative surveys by Statistics Canada designed to permit comparisons between populations and trends over time. Surveys of the general population were conducted in 2002 ( $n=$ 25643 ) and 2012 ( $n=15981$ ); those of military personnel were conducted in $2002(n=5153)$ and $2013(n=6700)$. We assessed the lifetime and past-year prevalence of suicidal ideation, plans and attempts, as well as use of mental health services.

Results: In 2012/13, but not in 2002, military personnel had significantly higher odds of both lifetime and past-year suicidal ideation than the civilian population (lifetime: adjusted odds ratio [OR] 1.32, 95\% confidence interval [CI] 1.171.50; past year: adjusted OR $1.34,95 \% \mathrm{Cl} 1.09-$ 1.66). The same was true for suicidal plans (lifetime: adjusted OR 1.64, 95\% Cl 1.35-1.99; past year: adjusted OR 1.66, 95\% Cl 1.18-2.33). Among respondents who reported past-year suicidal ideation, those in the military had a significantly higher past-year utilization rate of mental health services than those in the civilian population in both 2002 (adjusted OR 2.02, 95\% Cl 1.31-3.13) and 2012/13 (adjusted OR $3.14,95 \%$ Cl 1.86-5.28).

Interpretation: Canadian Forces personnel had a higher prevalence of suicidal ideation and plans in 2012/13 and a higher use of mental health services in 2002 and 2012/13 than the civilian population.
\end{abstract}

Competing interests: None declared.

This article has been peer reviewed.

Accepted: Feb. 22, 2016

Online: May 24, 2016

Correspondence to:

Jitender Sareen,

sareen@cc.umanitoba.ca

CMAJ 2016. DOI:10.1503/ cmaj.151047
$\mathrm{S}$ uicide is a leading cause of death around the world in military and civilian populations. $^{1-3}$ There has been increased attention paid to suicidal behaviour in Canada, and a number of initiatives are being put in place to prevent suicide through better recognition and treatment of mental disorders. ${ }^{4}$ Examples of major Canadian initiatives include creation of a national Mental Health Commission of Canada, ${ }^{5}$ development of a federal framework for suicide prevention, ${ }^{6}$ large investments in military and veteran mental health services, and targeted efforts to formulate comprehensive suicide prevention strategies among military and veteran populations. ${ }^{4,7}$ Despite these initiatives, the prevalence of suicide in Canada has not changed appreciably in recent years. ${ }^{8,9}$

A recent report on suicides in the Canadian Forces did not find an overall increase in the prevalence of suicide between 1995 and 2014. ${ }^{10}$ However, the prevalence increased substantially over that time in the subgroup of male army per- sonnel in the Regular Force. ${ }^{10}$ In the United States, the army has observed steady increases in the prevalence of suicide attempts and completed suicide by soldiers since 2004, whereas the prevalence of suicide has remained unchanged in the general population. ${ }^{3,11,12}$ Findings from the US are not generalizable to the Canadian military because of differences in recruitment, deployment policies and health care systems. ${ }^{13}$

Suicidal ideation, plans and attempts are strong risk factors for death by suicide. ${ }^{14} \mathrm{~A}$ history of suicide attempt is the strongest predictor of future attempts. ${ }^{15}$ Suicidal ideation is also an important target for intervention because previous work has shown a rapid transition from first-onset suicidal ideation to plans and attempts within the same year. ${ }^{16}$ It remains unknown whether nonfatal suicidal behaviour in military and civilian populations in Canada has changed over time.

Another area of major public health concern is that most people with suicidal behaviour do not 
receive mental health services. In nationally representative civilian samples in Canada and 21 other countries, most respondents with suicidal behaviours $(60 \%)$ did not receive mental health services. ${ }^{17,18}$ The use of such services among Canadian military personnel with suicidal behaviours remains unknown. The media has recently been critical of the Canadian Armed Forces and Veterans Affairs Canada about insufficient services available to military personnel and veterans. ${ }^{19}$

We compared trends in the prevalence of suicidal behaviours and help-seeking between Canadian civilian and military populations over a 10-year period from 2002 to 2012/13 using data from 4 nationally representative surveys.

\section{Methods}

\section{Data sources}

Data were obtained from 4 nationally representative surveys conducted by Statistics Canada in 2002 and 2012/13. For the civilian population, we used data from the 2002 Canadian Community Health Survey (cycle 1.2; $n=36$ 984, response rate $77.0 \%$ ) and the 2012 Canadian Community Health Survey - Mental Health $(n=$ 25113 , response rate $68.9 \%$ ). For the military population, we obtained data from the Canadian Forces Supplement of the 2002 Canadian Community Health Survey $(n=8441$, response rate $81.1 \%$ ) and the 2013 Canadian Forces Mental Health Survey ( $n=8393$, response rate 79.8\%).

Sampling frames to ensure representativeness of populations were used across all 4 surveys. Data were collected through face-to-face interviews by trained lay interviewers using computerassisted interviewing techniques. Participation in each of the surveys was voluntary, and consent was obtained before conducting each survey. Respondents' privacy and confidentiality was ensured based on the Statistics Act. Details of the 4 surveys have been published elsewhere. ${ }^{20-22}$

\section{Study population}

We restricted our analyses to respondents 18-60 years of age to maintain age comparability across the surveys. In addition, we excluded Reserve Force members from the military samples because the sampling design differed between the 2002 and 2013 surveys. In 2002, the Reserve Force segment was a representative sample, whereas in 2013, it included only Reserve Force members who had deployed in support of the mission in Afghanistan. The total sample in the merged data set across the 4 samples was 53477 (civilian: $n=25643$ in 2002 and 15981 in 2012; military: $n=5153$ in 2002 and 6700 in 2013).

\section{Outcome measures}

Suicidal ideation, plans and attempts were assessed through a series of questions. Respondents were asked if they had (a) seriously thought about committing suicide or taking their own life; (b) made a plan for committing suicide; or (c) attempted suicide or tried to take their own life. These behaviours were assessed for lifetime and past-year time frames. Suicidal plans were not assessed in the 2002 surveys; therefore, we compared this behaviour only for the 2012/13 survey cycles.

Past-year use of mental health services was assessed through a series of questions about contact with health care professionals for problems with emotions, mental health, or use of alcohol or drugs. Health care professionals included psychiatrists; psychologists; family physicians or general practitioners; nurses; and social workers, counsellors or psychotherapists. Dichotomous assessments were made for each category of health care professional seen in the past year separately (yes or no). Separate variables were computed for whether the respondent had contact with any health care professional in the past year (yes or no) and the total number of professionals seen in the past year ( 0 to 5 ).

\section{Sociodemographic covariates}

We included the following sociodemographic variables as covariates in the models: age (18$29,30-39,40-60$ yr), sex, visible minority status (yes or no), education (high school or less, some postsecondary, or university degree or higher) and income $(<\$ 80000$ or $\geq \$ 80000)$.

\section{Statistical analysis}

Statistical weights supplied by Statistics Canada were applied to the data to ensure that estimates were representative of each respective population. Bootstrapping was performed as a variance estimation technique with the use of STATA software to account for the complex survey designs. First, we computed overall and sexspecific prevalence estimates for lifetime and past-year suicidal behaviours across the 4 surveys. A series of multivariable logistic regression models, adjusted for sociodemographic covariates, were computed to test for differences in the prevalence of lifetime and past-year suicidal behaviours across the 4 surveys in the merged data set.

Second, we computed a series of multivariable logistic regression models, adjusted for sociodemographic covariates, to test for differences in the prevalence of past-year treatment-seeking among respondents who reported each type of suicidal behaviour across the 4 surveys in the merged data set. 
Third, we calculated estimated marginal means for the total number of health care professionals seen in the past year by computing coefficients derived from a negative binomial regression model adjusted for sociodemographic variables. We calculated differences in estimated marginal means across the surveys using these coefficients.

Fourth, interaction terms for time (2002 v. 2012/13) and population (civilian v. military) were entered into multivariate models to test whether the changes in all outcomes (i.e., suicidal behaviours and use of mental health services) among military personnel were greater than those in the general population.

Finally, we conducted a post-hoc analysis to examine whether the observed decrease in lifetime suicidal ideation among female military personnel from 2002 to 2013 was due to a decrease in combat exposure. Combat exposure was assessed with an item that asked whether the respondent had ever participated in combat, either as a member of the military or as a member of an organized nonmilitary group. Cross-tabulations were used to examine the prevalence of combat exposure by sex in the 2002 and 2013 military samples.

\section{Results}

The lifetime and past-year prevalence of suicidal behaviours in the 4 samples are shown in Tables 1 and 2 . We found few differences in the prevalence of suicidal behaviours between the military and civilian populations in 2002. In 2012/13, military personnel had significantly higher odds of both lifetime and past-year suicidal ideation than the civilian population (lifetime: adjusted odds ratio [OR] 1.32, 95\% confidence interval [CI] 1.171.50; past year: adjusted OR 1.34, 95\% CI 1.09 1.66). The same was true for suicidal plans (lifetime: adjusted OR 1.64, 95\% CI 1.35-1.99; past year: adjusted OR 1.66, 95\% CI 1.18-2.33). The time:population interaction terms showed that changes in lifetime suicidal ideation among male respondents were significantly greater in the military sample than in the civilian sample (adjusted OR $1.27,95 \%$ CI 1.05-1.53).

As shown in Table 1, there was a significant increase in the lifetime prevalence of suicide attempts among male military personnel from 2002 to 2013 (adjusted OR 1.51, 95\% CI 1.092.09 , which is reflected in an adjusted OR for the military population as a whole of $1.32,95 \% \mathrm{CI}$ 1.02-1.72). There was no significant change in the lifetime prevalence of suicide attempts over the same period among civilians. Among female military personnel, the lifetime prevalence of suicidal ideation decreased significantly over the 10-year period (adjusted OR 0.78, 95\% CI 0.62 0.97). There was no significant change in the lifetime prevalence of suicidal ideation over the same period among female civilians.

The use of mental health services among respondents who reported suicidal behaviours is

Table 1: Lifetime prevalence of suicidal behaviour among Canadian military personnel and civilians, 2002-2012/13

\begin{tabular}{|c|c|c|c|c|c|c|c|c|}
\hline \multirow[b]{2}{*}{$\begin{array}{l}\text { Suicidal } \\
\text { behavior }\end{array}$} & \multicolumn{3}{|c|}{ Military personnel } & \multicolumn{3}{|c|}{ Civilian population } & \multicolumn{2}{|c|}{ Military v. civilian } \\
\hline & $\begin{array}{c}2002 \\
n=5153\end{array}$ & $\begin{array}{c}2013^{*} \\
n=6700\end{array}$ & $\begin{array}{c}\text { Adjusted ORt } \\
(95 \% \mathrm{Cl}) \\
2013 \text { v. } 2002\end{array}$ & $\begin{array}{c}2002 \\
n=25643\end{array}$ & $\begin{array}{c}2012 \\
n=15981\end{array}$ & $\begin{array}{c}\text { Adjusted ORT } \\
(95 \% \text { Cl), } \\
2012 \text { v. } 2002\end{array}$ & $\begin{array}{l}\text { Adjusted OR† } \\
(95 \% \mathrm{Cl}) \\
2002\end{array}$ & $\begin{array}{c}\text { Adjusted OR }{ }^{-} \\
(95 \% \mathrm{Cl}) \\
2012 / 13\end{array}$ \\
\hline \multicolumn{9}{|l|}{ All } \\
\hline Ideation & $15.7(14.7-16.9)$ & $15.4(14.5-16.3)$ & $1.04(0.93-1.17)$ & $15.2(14.6-15.8)$ & $13.3(12.5-14.1)$ & $0.93(0.85-1.02)$ & $1.09(0.98-1.22)$ & $1.32(1.17-1.50)$ \\
\hline Attempt & $2.5(2.1-3.1)$ & $3.0(2.5-3.5)$ & $1.32(1.02-1.72)$ & $3.6(3.3-3.9)$ & $3.5(3.1-4.0)$ & $1.10(0.93-1.30)$ & $0.92(0.74-1.15)$ & $1.14(0.91-1.44)$ \\
\hline \multicolumn{9}{|l|}{ Men } \\
\hline Ideation & $14.8(13.6-16.0)$ & $14.9(13.9-15.9)$ & $1.11(0.97-1.26)$ & $14.0(13.2-14.9)$ & $11.8(10.7-12.9)$ & $0.88(0.77-1.00)$ & $1.03(0.91-1.18)$ & $1.30(1.12-1.52)$ \\
\hline Plan & NA & $5.9(5.3-6.6)$ & NA & NA & $4.0(3.5-4.7)$ & NA & NA & $1.66(1.33-2.08)$ \\
\hline Attempt & $2.1(1.6-2.7)$ & $2.6(2.1-3.1)$ & $1.51(1.09-2.09)$ & $2.4(2.1-2.7)$ & $2.7(2.2-3.2)$ & $1.23(0.98-1.54)$ & $0.83(0.62-1.11)$ & $1.15(0.85-1.55)$ \\
\hline Attempt & $5.8(4.7-7.0)$ & $5.4(3.9-7.5)$ & $0.92(0.59-1.44)$ & $4.8(4.4-5.3)$ & $4.4(3.7-5.2)$ & $1.03(0.82-1.29)$ & $1.22(0.93-1.61)$ & $1.42(0.93-2.18)$ \\
\hline $\begin{array}{l}\text { Note: } \mathrm{Cl}= \\
{ }^{*} \text { In the Car } \\
\text { Statistics C } \\
\text { tAdjusted }\end{array}$ & $\begin{array}{l}\text { fidence interva } \\
\text { ian Forces } 201 \\
\text { da's data-relea } \\
\text { age, sex, marit }\end{array}$ & $\begin{array}{l}\mathrm{A}=\text { not availabl } \\
\text { vey, percentage } \\
\text { olicies. } \\
\text { atus, visible mir }\end{array}$ & $\begin{array}{l}\text { data set or } n \\
\text { ere based on }\end{array}$ & $\begin{array}{l}\text { eased by Stat } \\
\text { ted numbers }\end{array}$ & $\begin{array}{l}\text { Canada to pro } \\
\text { h were round }\end{array}$ & $\begin{array}{l}\text { spondents' } \\
\text { a base } 20 \text { fo }\end{array}$ & $\begin{array}{l}\text { dentiality, } O R= \\
\text { fidentiality pur }\end{array}$ & $\begin{array}{l}\text { ds ratio. } \\
\text { es according to }\end{array}$ \\
\hline
\end{tabular}


shown in Table 3. Among those who reported past-year suicidal ideation, the prevalence of help-seeking increased significantly over the 10 -year study period in both the civilian and the military groups. The use of mental health services was significantly higher among the military personnel than among the civilians who reported past-year suicidal ideation in both 2002 (adjusted OR 2.02, 95\% CI 1.31-3.13) and 2012/13 (adjusted OR 3.14, 95\% CI 1.86-5.28). The time:population interaction terms were not significant in the help-seeking models.

In the post-hoc analysis (data available on request), the decrease in lifetime suicidal ideation observed among female military personnel over the study period was not attributable to a decrease in combat exposure. Both male and female military personnel had increases in combat exposure over the 10-year period (from 9\% to $20 \%$ among women and from $21 \%$ to $37 \%$ among men in the military).

\section{Interpretation}

Our study provides information on national trends in suicidal behaviour and use of mental health services in Canadian military and civilian populations. In the 2012/13 survey period, military personnel had a significantly higher prevalence of suicidal ideation and plans than the civilian population. The lifetime prevalence of suicide attempts increased significantly among military personnel, with no significant change over the same period among civilians. The prevalence of lifetime and past-year suicidal ideation among male military personnel did not change over time; however, female military personnel had a significant decrease in lifetime suicidal ideation. Finally, among people who reported suicidal behaviours, military personnel had a significantly higher prevalence of help-seeking and number of professionals seen than civilians.

Our findings of a higher prevalence of suicidal ideation and plans among military personnel than among civilians in the recent survey period, and an increase in the lifetime prevalence of suicide attempts among men in the military over time, are consistent with previous studies in Canada. ${ }^{10}$ They are also consistent with studies in the US, where increasing trends in suicide attempts and deaths have been observed. . $^{23,24}$

There are several possible explanations for our findings. First, the increase in suicidal behaviour may have been related to a concurrent increase in the prevalence of posttraumatic stress disorder and other anxiety disorders over time among men in the military. ${ }^{7,25,26}$ Second, a greater proportion of military personnel may have had increased exposure to traumatic experiences that are more strongly linked to suicidal behaviour during their deployments compared with the military personnel in the 2002 survey. ${ }^{27}$ Among American soldiers, preenlistment suicidal behaviour is common. ${ }^{3}$ The prevalence of pre-enlistment suicidal behaviour

Table 2: Past-year prevalence of suicidal behaviour among Canadian military personnel and civilians, 2002-2012/13

\begin{tabular}{|c|c|c|c|c|c|c|c|c|}
\hline \multirow[b]{3}{*}{$\begin{array}{l}\text { Suicidal } \\
\text { behavior }\end{array}$} & \multicolumn{3}{|c|}{ Military personnel } & \multicolumn{3}{|c|}{ Civilian population } & \multicolumn{2}{|c|}{ Military v. civilian } \\
\hline & \multicolumn{2}{|c|}{ Prevalence, \% $(95 \% \mathrm{Cl})$} & \multirow{2}{*}{$\begin{array}{l}\text { Adjusted OR† } \\
(95 \% \mathrm{Cl}) \\
2013 \text { v. } 2002\end{array}$} & \multicolumn{2}{|c|}{ Prevalence, $\%(95 \% \mathrm{Cl})$} & \multirow{2}{*}{$\begin{array}{c}\text { Adjusted OR } \dagger \\
(95 \% \mathrm{Cl}) \\
2012 \text { v. } 2002\end{array}$} & \multirow{2}{*}{$\begin{array}{c}\text { Adjusted OR } \dagger \\
(95 \% \mathrm{Cl}) \\
2002\end{array}$} & \multirow{2}{*}{$\begin{array}{c}\text { Adjusted OR }{ }^{-} \\
(95 \% \mathrm{Cl}) \\
2012 / 13\end{array}$} \\
\hline & $\begin{array}{c}2002 \\
n=5153\end{array}$ & $\begin{array}{c}2013^{*} \\
n=6700\end{array}$ & & $\begin{array}{c}2002 \\
n=25643\end{array}$ & $\begin{array}{c}2012 \\
n=15981\end{array}$ & & & \\
\hline \multicolumn{9}{|l|}{ All } \\
\hline Ideation & $4.2(3.7-4.8)$ & $4.3(3.7-4.9)$ & $1.13(0.91-1.40)$ & $3.9(3.6-4.3)$ & $3.6(3.2-4.1)$ & $1.00(0.84-1.19)$ & $1.11(0.92-1.33)$ & $1.34(1.09-1.66)$ \\
\hline Plan & NA & $1.8(1.5-2.1)$ & NA & NA & $1.3(1.0-1.7)$ & NA & NA & $1.66(1.18-2.33)$ \\
\hline Attempt & $0.3(0.2-0.5)$ & $0.4(0.2-0.6)$ & $1.33(0.61-2.87)$ & $0.6(0.5,0.7)$ & $0.6(0.4-0.9)$ & $1.29(0.78-2.14)$ & $0.61(0.34-1.08)$ & $0.88(0.43-1.77)$ \\
\hline \multicolumn{9}{|l|}{ Men } \\
\hline Ideation & $4.0(3.4-4.7)$ & $4.3(3.7-4.9)$ & $1.17(0.92-1.49)$ & $4.0(3.5-4.5)$ & $3.4(2.9-4.0)$ & $0.90(0.71-1.16)$ & $1.11(0.90-1.38)$ & $1.43(1.10-1.86)$ \\
\hline Plan & NA & $1.8(1.5-2.2)$ & NA & NA & $1.3(0.9-1.7)$ & NA & NA & $1.84(1.22-2.77)$ \\
\hline Attempt & $0.2(0.1-0.4)$ & NA & NA & $0.5(0.3-0.6)$ & $0.5(0.3-0.7)$ & $1.11(0.62-2.02)$ & $0.42(0.15-1.18)$ & NA \\
\hline \multicolumn{9}{|l|}{ Women } \\
\hline Ideation & $5.3(4.3-6.5)$ & $4.3(2.9-6.0)$ & $0.93(0.55-1.56)$ & $3.9(3.4-4.4)$ & $3.9(3.2-4.5)$ & $1.09(0.86-1.39)$ & $1.41(1.07-1.85)$ & $1.17(0.75-1.83)$ \\
\hline Plan & NA & $1.6(0.8-2.8)$ & NA & NA & $1.3(0.9-2.0)$ & NA & NA & $1.23(0.55-2.76)$ \\
\hline Attempt & $1.1(0.7-1.8)$ & NA & NA & $0.7(0.5-0.9)$ & $0.7(0.4-1.3)$ & $1.40(0.67-2.89)$ & $1.69(0.85-3.36)$ & NA \\
\hline
\end{tabular}


Table 3: Prevalence of past-year help-seeking among Canadian military and civilians who reported past-year suicidal behaviours

\begin{tabular}{|c|c|c|c|c|c|c|c|c|}
\hline \multirow{3}{*}{$\begin{array}{l}\text { Sector of } \\
\text { treatment; } \\
\text { reported } \\
\text { behaviour }\end{array}$} & \multicolumn{3}{|c|}{ Military personnel } & \multicolumn{3}{|c|}{ Civilian population } & \multicolumn{2}{|c|}{ Military v. civilian } \\
\hline & \multicolumn{2}{|c|}{ Prevalence, $\%(95 \% \mathrm{Cl})$} & \multirow{2}{*}{$\begin{array}{c}\text { Adjusted OR† } \\
(95 \% \mathrm{Cl}) \\
2013 \text { v. } 2002\end{array}$} & \multicolumn{2}{|c|}{ Prevalence, $\%(95 \% \mathrm{Cl})$} & \multirow{2}{*}{$\begin{array}{l}\text { Adjusted OR† } \\
(95 \% \mathrm{Cl}) \\
2012 \text { v. } 2002\end{array}$} & \multirow{2}{*}{$\begin{array}{l}\text { Adjusted OR } t \\
(95 \% \mathrm{Cl}), \\
2002\end{array}$} & \multirow{2}{*}{$\begin{array}{c}\text { Adjusted ORt } \\
(95 \% \mathrm{Cl}) \\
2012 / 13\end{array}$} \\
\hline & 2002 & $2013^{*}$ & & 2002 & 2012 & & & \\
\hline \multicolumn{9}{|l|}{ Psychiatrist } \\
\hline Ideation & $\begin{array}{c}24.6 \\
(18.6-31.8)\end{array}$ & $\begin{array}{c}38.7 \\
(32.3-45.4)\end{array}$ & $\begin{array}{c}2.32 \\
(1.42-3.77)\end{array}$ & $\begin{array}{c}17.3 \\
(14.5-20.5)\end{array}$ & $\begin{array}{c}20.9 \\
(16.9-25.6)\end{array}$ & $\begin{array}{c}1.45 \\
(1.00-2.09)\end{array}$ & $\begin{array}{c}1.73 \\
(1.03-2.92)\end{array}$ & $\begin{array}{c}2.41 \\
(1.39-4.17)\end{array}$ \\
\hline Plan & NA & $\begin{array}{c}45.6 \\
(36.3-56.9)\end{array}$ & NA & NA & $\begin{array}{c}35.7 \\
(25.3-47.7)\end{array}$ & NA & NA & $\begin{array}{c}1.18 \\
(0.41-3.46)\end{array}$ \\
\hline Attempt & $\begin{array}{c}47.2 \\
(26.6-68.9)\end{array}$ & $\begin{array}{c}50.0 \\
(28.9-74.4)\end{array}$ & $\begin{array}{c}1.32 \\
(0.11-16.11)\end{array}$ & $\begin{array}{c}37.6 \\
(28.1-48.2)\end{array}$ & $\begin{array}{c}30.1 \\
(16.7-47.9)\end{array}$ & $\begin{array}{c}0.73 \\
(0.31-1.72)\end{array}$ & $\begin{array}{c}1.64 \\
(0.49-5.45)\end{array}$ & $\begin{array}{c}3.93 \\
(0.84-18.39)\end{array}$ \\
\hline \multicolumn{9}{|l|}{ Psychologist } \\
\hline Ideation & $\begin{array}{c}25.4 \\
(19.4-32.6)\end{array}$ & $\begin{array}{c}40.9 \\
(34.4-47.6)\end{array}$ & $\begin{array}{c}2.17 \\
(1.35-3.48)\end{array}$ & $\begin{array}{c}12.0 \\
(9.5-15.2)\end{array}$ & $\begin{array}{c}17.5 \\
(12.6-23.9)\end{array}$ & $\begin{array}{c}1.49 \\
(0.95-2.35)\end{array}$ & $\begin{array}{c}2.42 \\
(1.26-4.65)\end{array}$ & $\begin{array}{c}4.67 \\
(2.58-8.47)\end{array}$ \\
\hline Plan & NA & $\begin{array}{c}50.9 \\
(40.1-61.2)\end{array}$ & NA & NA & $\begin{array}{c}28.2 \\
(16.8-43.2)\end{array}$ & NA & NA & $\begin{array}{c}3.88 \\
(0.94-16.04)\end{array}$ \\
\hline Attempt & $\begin{array}{c}48.3 \\
(27.2-70.1)\end{array}$ & $\begin{array}{c}50.0 \\
(29.3-72.3)\end{array}$ & $\begin{array}{c}0.94 \\
(0.15-5.74)\end{array}$ & $\begin{array}{c}17.4 \\
(9.9-28.6)\end{array}$ & $\begin{array}{c}32.0 \\
(14.0-57.6)\end{array}$ & $\begin{array}{c}2.37 \\
(0.55-10.23)\end{array}$ & $\begin{array}{c}4.58 \\
(1.15-18.15)\end{array}$ & $\begin{array}{c}6.33 \\
(0.65-61.56)\end{array}$ \\
\hline \multicolumn{9}{|l|}{ Family physician } \\
\hline Ideation & $\begin{array}{c}36.0 \\
(29.2-43.4)\end{array}$ & $\begin{array}{c}43.8 \\
(36.8-50.6)\end{array}$ & $\begin{array}{c}1.48 \\
(0.94-2.34)\end{array}$ & $\begin{array}{c}31.2 \\
(27.1-35.6)\end{array}$ & $\begin{array}{c}40.0 \\
(33.4-46.9)\end{array}$ & $\begin{array}{c}1.53 \\
(1.09-2.15)\end{array}$ & $\begin{array}{c}1.94 \\
(1.23-3.05)\end{array}$ & $\begin{array}{c}1.71 \\
(1.02-2.84)\end{array}$ \\
\hline Plan & NA & $\begin{array}{c}49.1 \\
(39.8-59.9)\end{array}$ & NA & NA & $\begin{array}{c}57.4 \\
(45.0-68.9)\end{array}$ & NA & NA & $\begin{array}{c}1.01 \\
(0.33-3.08)\end{array}$ \\
\hline Attempt & $\begin{array}{c}60.7 \\
(37.6-79.8)\end{array}$ & $\begin{array}{c}58.3 \\
(35.2-77.1)\end{array}$ & $\begin{array}{c}1.18 \\
(0.14-10.14)\end{array}$ & $\begin{array}{c}41.2 \\
(31.3-51.8)\end{array}$ & $\begin{array}{c}70.8 \\
(53.9-83.4)\end{array}$ & $\begin{array}{c}3.55 \\
(1.45-8.69)\end{array}$ & $\begin{array}{c}2.68 \\
(0.69-10.38)\end{array}$ & $\begin{array}{c}0.55 \\
(0.13-2.39)\end{array}$ \\
\hline \multicolumn{9}{|l|}{ Nurse } \\
\hline Ideation & $\begin{array}{c}14.9 \\
(10.2-21.2)\end{array}$ & $\begin{array}{c}29.2 \\
(23.6-35.0)\end{array}$ & $\begin{array}{c}2.84 \\
(1.59-5.09)\end{array}$ & $\begin{array}{c}4.4 \\
(3.1-6.1)\end{array}$ & $\begin{array}{c}7.5 \\
(5.3-10.5)\end{array}$ & $\begin{array}{c}1.77 \\
(1.03-3.06)\end{array}$ & $\begin{array}{c}7.51 \\
(3.13-18.06)\end{array}$ & $\begin{array}{c}6.54 \\
(3.00-14.28)\end{array}$ \\
\hline Plan & NA & $\begin{array}{c}40.4 \\
(31.5,50.8)\end{array}$ & NA & NA & $\begin{array}{c}10.8 \\
(6.4-17.6)\end{array}$ & NA & NA & $\begin{array}{c}8.53 \\
(1.95-37.25)\end{array}$ \\
\hline Attempt & $\begin{array}{c}35.0 \\
(17.5-57.8)\end{array}$ & $\begin{array}{c}58.3 \\
(33.6-76.9)\end{array}$ & $\begin{array}{c}2.48 \\
(0.33-18.98)\end{array}$ & $\begin{array}{c}12.2 \\
(7.0-20.5)\end{array}$ & $\begin{array}{c}11.0 \\
(5.4-21.3)\end{array}$ & $\begin{array}{c}0.93 \\
(0.35-2.47)\end{array}$ & $\begin{array}{c}5.26 \\
(1.31-21.15)\end{array}$ & $\begin{array}{c}23.40 \\
(1.86-294.48)\end{array}$ \\
\hline \multicolumn{9}{|l|}{ Social worker } \\
\hline Ideation & $\begin{array}{c}25.4 \\
(19.1-33.0)\end{array}$ & $\begin{array}{c}52.6 \\
(45.8-58.7)\end{array}$ & $\begin{array}{c}3.76 \\
(2.29-6.16)\end{array}$ & $\begin{array}{c}11.6 \\
(8.6-15.5)\end{array}$ & $\begin{array}{c}22.5 \\
(18.2-27.5)\end{array}$ & $\begin{array}{c}2.43 \\
(1.54-3.86)\end{array}$ & $\begin{array}{c}4.17 \\
(2.20-7.91)\end{array}$ & $\begin{array}{c}4.84 \\
(2.83-8.27)\end{array}$ \\
\hline Plan & NA & $\begin{array}{c}54.4 \\
(43.8-64.0)\end{array}$ & NA & NA & $\begin{array}{c}29.0 \\
(20.5-39.3)\end{array}$ & NA & NA & $\begin{array}{c}4.23 \\
(1.39-12.85)\end{array}$ \\
\hline Attempt & $\begin{array}{c}39.5 \\
(20.6-62.2)\end{array}$ & NA & NA & $\begin{array}{c}17.1 \\
(11.2-25.2)\end{array}$ & $\begin{array}{c}30.6 \\
(17.6-47.7)\end{array}$ & $\begin{array}{c}2.19 \\
(0.78-6.17)\end{array}$ & $\begin{array}{c}3.60 \\
(1.08-12.02)\end{array}$ & NA \\
\hline \multicolumn{9}{|c|}{ Any treatment seeking } \\
\hline Ideation & $\begin{array}{c}51.3 \\
(44.0-58.6)\end{array}$ & $\begin{array}{c}73.0 \\
(66.6-78.6)\end{array}$ & $\begin{array}{c}3.16 \\
(1.96-5.08)\end{array}$ & $\begin{array}{c}42.1 \\
(37.6-46.8)\end{array}$ & $\begin{array}{c}57.0 \\
(50.8-63.0)\end{array}$ & $\begin{array}{c}1.90 \\
(1.37-2.62)\end{array}$ & $\begin{array}{c}2.02 \\
(1.31-3.13)\end{array}$ & $\begin{array}{c}3.14 \\
(1.86-5.28)\end{array}$ \\
\hline Plan & NA & $\begin{array}{c}77.2 \\
(68.1-85.7)\end{array}$ & NA & NA & $\begin{array}{c}75.7 \\
(66.0-83.3)\end{array}$ & NA & NA & $\begin{array}{c}1.84 \\
(0.61-5.53)\end{array}$ \\
\hline Attempt & $\begin{array}{c}72.0 \\
(47.5-87.9)\end{array}$ & NA & NA & $\begin{array}{c}58.4 \\
(46.8-69.1)\end{array}$ & $\begin{array}{c}79.8 \\
(65.0-89.4)\end{array}$ & $\begin{array}{c}2.83 \\
(1.13-7.11)\end{array}$ & $\begin{array}{c}2.00 \\
(0.49-8.22)\end{array}$ & NA \\
\hline \multirow{2}{*}{$\begin{array}{l}\text { No. of } \\
\text { professionals } \neq\end{array}$} & \multicolumn{2}{|c|}{ Mean no. $(95 \% \mathrm{Cl})$} & & \multicolumn{2}{|c|}{ Mean no. $(95 \% \mathrm{Cl})$} & & & \\
\hline & 2002 & $2013^{*}$ & $\chi^{2}$ & 2002 & 2012 & $\chi^{2}$ & $\chi^{2}$ & $\chi^{2}$ \\
\hline Ideation & $\begin{array}{c}1.5 \\
(1.2-1.8)\end{array}$ & $\begin{array}{c}2.6 \\
(2.1-3.0)\end{array}$ & 21.46 & $\begin{array}{c}0.7 \\
(0.6-0.8)\end{array}$ & $\begin{array}{c}1.1 \\
(1.0-1.2)\end{array}$ & 19.73 & 18.53 & 34.65 \\
\hline Plan & NA & $\begin{array}{c}2.5 \\
(1.8-3.3)\end{array}$ & NA & NA & $\begin{array}{c}1.6 \\
(1.3-1.9)\end{array}$ & NA & NA & 4.87 \\
\hline Attempt & $\begin{array}{c}2.5 \\
(1.4-3.5)\end{array}$ & $\begin{array}{c}3.5 \\
(2.5-4.5)\end{array}$ & 2.13 & $\begin{array}{c}1.2 \\
(0.9-1.5)\end{array}$ & $\begin{array}{c}1.7 \\
(1.4-2.1)\end{array}$ & 7.02 & 5.12 & 10.99 \\
\hline $\begin{array}{l}\text { Note: } \mathrm{Cl}=\text { confider } \\
\text { *In the Canadian } \\
\text { Statistics Canada's } \\
\text { †Suicide ideation } \\
\text { age and sex. } \\
\text { †Estimated margin }\end{array}$ & $\begin{array}{l}\text { erval, NA = } \\
013 \text { survey } \\
\text { elease polic } \\
\text { cide plan n } \\
\text { ins (i.e., ad }\end{array}$ & $\begin{array}{l}\text { vailable in o } \\
\text { entages wer } \\
\text { adjusted fo } \\
\text { means) we }\end{array}$ & $\begin{array}{l}\text { set or not relea } \\
\text { sed on weight } \\
\text { e, sex, marital }\end{array}$ & $\begin{array}{l}\text { by Statistics } \\
\text { umbers, wh } \\
\text { s, visible mi }\end{array}$ & $\begin{array}{l}\text { hada to prote } \\
\text { were rounded } \\
\text { ity status, edu }\end{array}$ & $\begin{array}{l}\text { spondents' c } \\
\text { a base } 20 \text { for } \\
\text { on and incon }\end{array}$ & $\begin{array}{l}\text { ntiality, OR = } \\
\text { entiality purp } \\
\text { ide attempt n }\end{array}$ & $\begin{array}{l}\text { s ratio. } \\
\text { s according to } \\
\text { els adjusted for }\end{array}$ \\
\hline
\end{tabular}


(and related risk factors for suicidality) among Canadian soldiers may have changed over time. However, there have not been relevant policy changes in the Canadian Forces to relax recruitment criteria over this period. Regardless of whether the causes of suicidal behaviour are related to pre-enlistment or deployment-related factors, trends of an increasing lifetime prevalence of suicide attempts over the 10-year period and of a higher prevalence of suicidal ideation and plans among military personnel than among civilians in 2012/13 is a concerning and important observation with public-policy ramifications.

The significant decrease in the prevalence of lifetime suicidal ideation among women in the military over the 10-year study period was unexpected. In post hoc analysis, we did not find that the decrease was due to lower combat exposure among the female respondents in the 2013 military sample. Both male and female military personnel had increases in combat exposure from 2002 to 2013. One possible explanation is that women are more likely than men to seek mental health services, and effective treatment of mental disorders is associated with lower rates of suicidal behaviour. ${ }^{28}$ Further examination of the reasons for the decrease in lifetime rates of suicidal ideation among female military personnel is required.

Although the Canadian Forces and Veterans Affairs Canada have been criticized strongly in the media about the concerns of lack of access to mental health services, ${ }^{19}$ in both survey periods, military personnel who reported suicidal behaviours were significantly more likely than their civilian counterparts to seek help. The Canadian health care system has been criticized for not being a universal health care system, with inequities in access, variation in service provision across provinces and substantial inefficiencies..$^{29}$ Our study supports this criticism by showing inequities in the receipt of mental health services between civilians and military personnel. Mental health services for military personnel are funded through a federally organized system, whereas civilians access care through a provincially funded system. ${ }^{29}$ This direct comparison between military and civilian samples suggests the need for stronger investment in mental health services for civilians such that there is equitable access to mental health services for civilians and military personnel. The Canadian military has also created post-deployment screening programs ${ }^{25}$ and anti-stigma campaigns so that personnel can access services in a timely manner. ${ }^{4}$ Certain highly stressful occupations such as firefighting and law enforcement may benefit from screening and antistigma campaigns similar to those used by the military.

\section{Limitations}

Our study has several limitations. First, we examined suicidal ideation, plans and attempts; therefore, findings are not generalizable to completed suicides. Second, recall errors may have biased the reporting of suicidal behaviour and help-seeking. Increased public attention on suicidal behaviour may have also influenced responses to suicide questions in recent surveys. Third, people with severe mental illness may have left military service or not participated in the general population surveys, such that the prevalence of suicidal behaviours we documented may be underestimated. Fourth, the 2013 survey of military personnel did not acquire a representative sample of Reserve Force members. Thus, we could not examine trends in suicidal behaviour among reservists. Fifth, the lethality of suicidal behaviour was not assessed in the surveys and may have differed over time. ${ }^{30}$ Nonetheless, the survey methodology was consistent across all 4 surveys and used stateof-the-art structured diagnostic interviews that are used around the world. ${ }^{31}$ Finally, our findings may not be generalizable to other countries with different health care systems and policies.

\section{Conclusion}

During the 10-year study period, the prevalence of suicidal ideation and plans, and the use of mental health services were higher among Canadian Forces personnel than among civilians. Male military personnel had a significant increase in the lifetime prevalence of suicide attempts, and female military personnel had a significant decrease in the lifetime prevalence of suicidal ideation. There were no significant trends in suicidal behaviour among civilians over the same 10-year period. Investments in clinical services and research are required to address suicidal behaviour among military and civilian populations.

\section{References}

1. Tien HC, Acharya S, Redelmeier DA. Preventing deaths in the Canadian military. Am J Prev Med 2010;38:331-9.

2. Hawton K, van Heeringen K. Suicide. Lancet 2009;373:1372-81.

3. Nock MK, Stein MB, Heeringa SG, et al. Prevalence and correlates of suicidal behavior among soldiers: results from the Army Study to Assess Risk and Resilience in Servicemembers (Army STARRS). JAMA Psychiatry 2014;71:514-22.

4. Zamorski MA. Suicide prevention in military organizations. Int Rev Psychiatry 2011;23:173-80.

5. Goldbloom DS. The Mental Health Commission of Canada is three years old: an update and reflection. Can J Nurs Res 2010;42:101-4.

6. Consultations on the development of a federal framework for suicide prevention. Ottawa: Public Health Agency of Canada; 2013. Available: www.phac-aspc.gc.ca/mh-sm/mhp-psm/new-nouv -eng.php (accessed 2015 Aug. 15).

7. Boulos D, Zamorski MA. Deployment-related mental disorders among Canadian Forces personnel deployed in support of the mission in Afghanistan, 2001-2008. CMAJ 2013;185:E545-52.

8. Rhodes AE, Skinner R, McFaull S, et al. Canada-wide effect of regulatory warnings on antidepressant prescribing and suicide rates in boys and girls. Can J Psychiatry 2013;58:640-5

9. Suicide and suicide prevention in the Canadian Armed Forces. Ottawa: National Defence and Canadian Armed Forces; 2014. 
Available: www.forces.gc.ca/en/news/article.page?doc=suicide -and-suicide-prevention-in-the-canadian-armed-forces/ijfc8m $4 \mathrm{~h}$ (accessed 2015 Aug. 15).

10. Rolland-Harris EW, Whitehead J, Matheson H, et al. 2015 report on suicide mortality in the Canadian Armed Forces (1995 to 2014). Ottawa: National Defence and the Canadian Armed Forces; 2015. Available: www.forces.gc.ca/en/about-reports-pubs-health/report -on-suicide-mortality-caf-2015.page (accessed 2015 Nov. 10).

11. Schoenbaum M, Kessler RC, Gilman SE, et al. Predictors of suicide and accident death in the Army Study to Assess Risk and Resilience in Servicemembers (Army STARRS): results from the Army Study to Assess Risk and Resilience in Servicemembers (Army STARRS). JAMA Psychiatry 2014;71:493-503.

12. LeardMann CA, Powell TM, Smith TC, et al. Risk factors associated with suicide in current and former US military personnel. JAMA 2013;310:496-506.

13. Belik SL, Stein MB, Asmundson GJ, et al. Are Canadian soldiers more likely to have suicidal ideation and suicide attempts than Canadian civilians? Am J Epidemiol 2010;172:1250-8.

14. Nock MK, Borges G, Bromet EJ, et al. Cross-national prevalence and risk factors for suicidal ideation, plans and attempts. Br J Psychiatry 2008;192:98-105.

15. Sareen J, Isaak C, Katz LY, et al. Promising strategies for advancement in knowledge of suicide risk factors and prevention. Am J Prev Med 2014;47(Suppl 2):S257-63.

16. Kessler RC, Borges G, Walters EE. Prevalence of and risk factors for lifetime suicide attempts in the National Comorbidity Survey. Arch Gen Psychiatry 1999;56:617-26.

17. Bruffaerts R, Demyttenaere K, Hwang I, et al. Treatment of suicidal people around the world. Br J Psychiatry 2011;199:64-70.

18. Pagura J, Fotti S, Katz LY, et al. Help seeking and perceived need for mental health care among individuals in Canada with suicidal behaviors. Psychiatr Serv 2009;60:943-9.

19. Mackenzie L. Canadian Forces: holding the line on mental health. Globe and Mail [Toronto]. 2014 Feb. 14

20. Sareen J, Cox BJ, Afifi TO, et al. Combat and peacekeeping operations in relation to prevalence of mental disorders and perceived need for mental health care: findings from a large representative sample of military personnel. Arch Gen Psychiatry 2007;64:843-52.

21. Gravel R, Beland Y. The Canadian Community Health Survey: mental health and well-being. Can J Psychiatry 2005;50:573-9.

22. Afifi TO, MacMillan HL, Boyle M, et al. Child abuse and mental disorders in Canada. CMAJ 2014;186:E324-32.

23. Reger MA, Smolenski DJ, Skopp NA, et al. Risk of suicide among US military service members following Operation Enduring Freedom or Operation Iraqi Freedom deployment and separation from the US military. JAMA Psychiatry 2015;72:561-9.

24. Pinder RJ, Iversen AC, Kapur N, et al. Self-harm and attempted suicide among UK armed forces personnel: results of a crosssectional survey. Int J Soc Psychiatry 2012;58:433-9.

25. Foran HM, Garber BG, Zamorski MA, et al. Postdeployment military mental health training: cross-national evaluations. Psychol Serv 2013;10:152-60.

26. Pearson C, Zamorski M, Janz T. Mental health of the Canadian Forces. Ottawa: Statistics Canada; 2014. Cat. no. 82-624-X.

27. Belik SL, Stein MB, Asmundson GJ, et al. Relation between traumatic events and suicide attempts in Canadian military personnel. Can J Psychiatry 2009;54:93-104.
28. Sareen J, Cox BJ, Afifi TO, et al. Combat and peacekeeping operations in relation to prevalence of mental disorders and perceived need for mental health care: findings from a large representative sample of military personnel. Arch Gen Psychiatry 2007;64:843-52.

29. Lewis S. A system in name only - access, variation, and reform in Canada's provinces. N Engl J Med 2015;372:497-500.

30. Nock MK, Kessler RC. Prevalence of and risk factors for suicide attempts versus suicide gestures: analysis of the National Comorbidity Survey. J Abnorm Psychol 2006;115:616-23.

31. Stein DJ, Chiu WT, Hwang I, et al. Cross-national analysis of the associations between traumatic events and suicidal behavior: findings from the WHO World Mental Health Surveys. PLoS One 2010;5:e10574.

Affiliations: Departments of Psychiatry (Sareen, Afifi, Bolton), Psychology (Sareen, Cheung, Erickson) and Community Health Sciences (Sareen, Afifi, Turner, Bolton), University of Manitoba, Winnipeg, Man.; Applied Health Sciences Program (Taillieu), Department of Psychiatry (Stein), University of California San Diego; VA San Diego Healthcare System (Stein), San Diego, Calif.; Defence Research and Development Canada (Fikretoglu); Directorate of Mental Health (Zamorski), Canadian Forces Health Services Group HQ; Department of Family Medicine, University of Ottawa, Ottawa, Ont.

Contributors: Jitender Sareen developed the research questions, designed and supervised the statistical analysis and interpreted the findings. Mark Zamorski and Tracie Afifi assisted with the development of the research questions and interpreted the findings. Tamara Taillieu, Kristene Cheung, Sarah Turner and Julie Erickson developed the research questions, and designed and conducted the statistical analysis. Shay-Lee Bolton provided feedback on the analytic approach, helped with the interpretation of findings and reviewed all analyses and drafts. Deniz Fikretoglu helped with the interpretation of findings on service use and provided suggestions for research questions and analytic approach. Murray Stein contributed to the research question and designed the statistical analysis. Jitender Sareen, Mark Zamorski and Tracie Afifi drafted the manuscript, and the remaining authors critically revised it for important intellectual content. All of the authors approved the final version to be published and agreed to act as guarantors of the work.

Funding: Preparation of this article was supported by a Canadian Institutes of Health Research New Investigator Award (Afifi) and a Manitoba Health Research Council Establishment Grant (Afifi)

Acknowledgement: Statistics Canada collected and provided the data for academic purposes, but the analyses are the sole responsibility of the authors. The opinions expressed do not represent the views of the Department of National Defence, the Canadian Forces or Statistics Canada. 